\title{
Top US universities, institutes for life sciences in 2015
}

\author{
Brady Huggett
}

$\mathrm{n}$ the basis of 5 years (2011-2015) of total gross licensing revenue reported by the Association of University Technology Managers (AUTM), Nature Biotechnology selected the top performers among universities and collected detailed life science commercialization information for 2015; those results are found in Table 1, re-ranked by numbers of licenses and/or options executed. Results for biomedical research institutes are in Table 2. The names in this top group of schools have

shifted over time, yet these metrics have been on a consistent upward trend, particularly so with startups: in 2013, the top schools collectively recorded 93 life science startups, compared to 182 in 2015. Life science revenue, licensing activity and startups account for the majority of overall totals for these schools (Fig. 1). New York University recorded the most in revenue, topping even the system-wide numbers of Texas and California (Fig. 2).

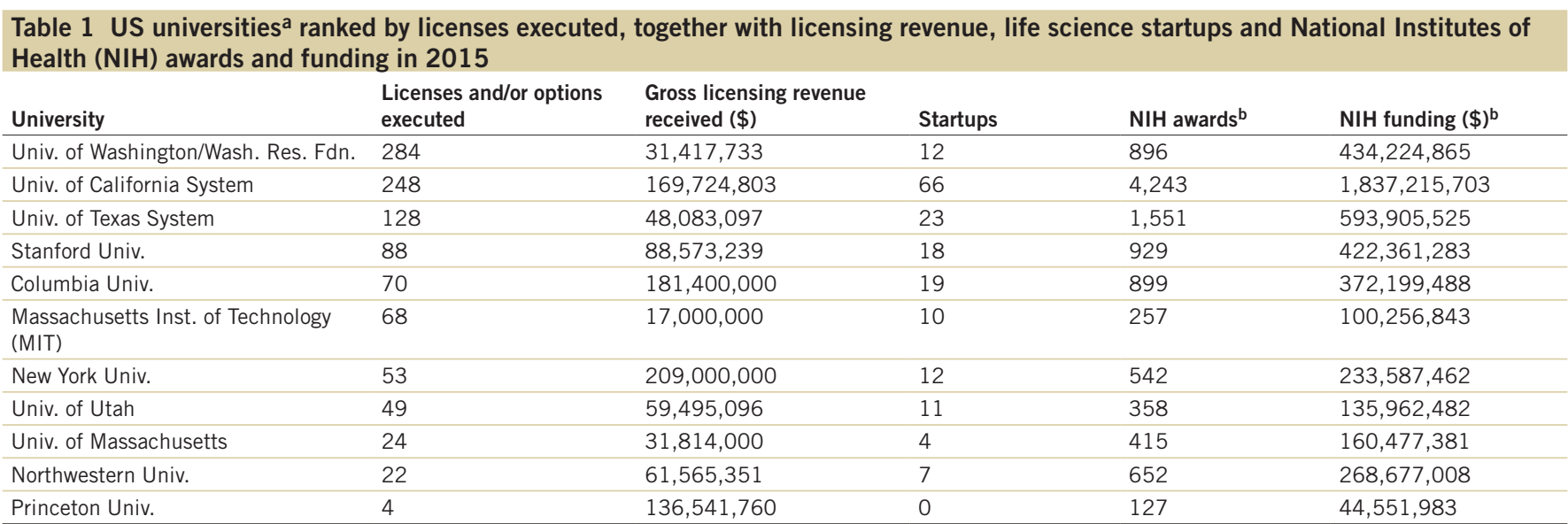

aThe University of Wisconsin-Madison also ranked highly in gross licensing revenue but could not provide information specific to life sciences. Source: AUTM, university technology transfer offices, NIH. ' NIH data for fiscal year 2015.

Table 2 US biomedical research institutes ranked by licenses and/or options executed, together with revenue, startups and NIH awards and funding in 2015

\begin{tabular}{|c|c|c|c|c|c|}
\hline Research institute & $\begin{array}{l}\text { Licenses and/or } \\
\text { options executed }\end{array}$ & $\begin{array}{l}\text { Gross licensing revenue } \\
\text { received }(\$)\end{array}$ & Startups & NIH awards ${ }^{a}$ & NIH funding ${ }^{a}$ \\
\hline $\begin{array}{l}\text { The General Hospital dba Massachusetts } \\
\text { General Hospital }\end{array}$ & 105 & $68,906,000$ & 6 & 776 & $350,015,302$ \\
\hline Mayo Fdn. for Medical Education and Research & 94 & $41,497,523$ & 7 & 401 & $201,234,282$ \\
\hline Brigham \& Women's Hospital Inc. & 67 & $9,375,921$ & 6 & 563 & $321,608,586$ \\
\hline Memorial Sloan Kettering Cancer Center & 42 & $164,769,901$ & 1 & 244 & $113,567,614$ \\
\hline Children's Hospital Boston & 33 & $7,328,686$ & 3 & 304 & $124,995,490$ \\
\hline Dana-Farber Cancer Inst. & 32 & $24,114,089$ & 0 & 210 & $122,813,873$ \\
\hline Beth Israel Deaconess Medical Center & 32 & $5,847,365$ & 4 & 240 & $108,156,442$ \\
\hline Cleveland Clinic & 28 & $16,246,038$ & 4 & 195 & $80,948,724$ \\
\hline The Salk Inst. for Biological Studies & 26 & $3,712,937$ & 2 & 79 & $40,510,332$ \\
\hline St. Jude Children's Research Hospital & 24 & $29,555,155$ & 0 & 90 & $62,482,117$ \\
\hline
\end{tabular}

Source: AUTM, NIH. ${ }^{\text {aNIH }}$ data from fiscal year 2015.

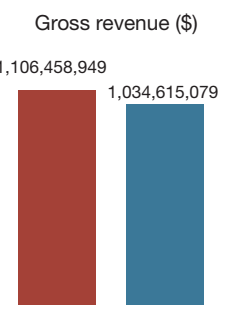

Overall
Licenses/options executed

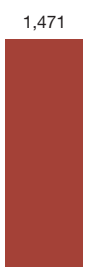

Overall

Life sciences

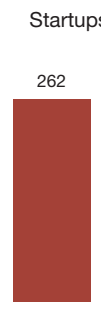

Overall

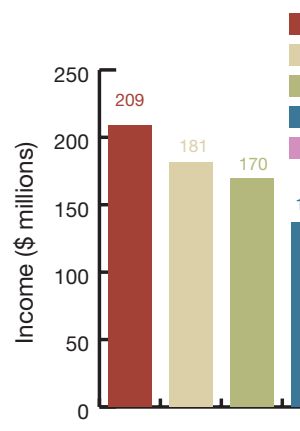

New York University Columbia University University of California System $\square$ University of Texas System Princeton University Stanford University

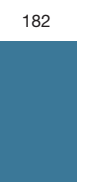

Life sciences

Figure 1 The life sciences accounted for the majority of incoming revenue, outgoing licenses and/or options and startup formation in 2015. Data from universities in Table 1. Source: AUTM, university technology transfer offices.

Figure 2 Life science licensing income earners, 2015. Source: AUTM, university technology transfer offices. 